\title{
RELACIÓN ENTRE LA COMPOSICIÓN DE ESPECIES DEL GÉNERO DE LUTZOMYIA FRANÇA (DIPTERA: PSYCHODIDAE, PHLEBOTOMINAE) Y LOS DIFERENTES TIPOS DE BOSQUES EN PANAMÁ
}

\author{
Anayansi Valderrama C.1, Martha Herrera ${ }^{2}$, Aleyda SAlazar 3 \\ 1 Sección de Entomología, Unidad de Investigaciones sobre enfermedades transmisibles \\ (Instituto Conmemorativo Gorgas) e-mail: anayvald@yahoo.com \\ 2 Facultad de Ciencia Naturales, Escuela de Biología, Universidad de Panamá, \\ 3 Autoridad Nacional del Ambiente.
}

\begin{abstract}
RESUMEN
Algunas especies del género Lutzomyia França son transmisores de la leishmaniasis cutánea y mucocutánea en Panamá. Su distribución está relacionada con la incidencia de casos en lugares rurales colindantes a zonas boscosas, sin embargo la presencia de los vectores puede registrarse en algunas zonas, sin que existan reportes de enfermos. Este estudio se realizó en las localidades de Gamboa, Altos de Campana y Altos Chagres, sitios ubicados dentro de la Cuenca del Canal de Panamá, durante los años del 2000 al 2001. Los ejemplares se capturaron con trampas CDC miniatura y se trasladaron al Instituto Conmemorativo Gorgas donde se realizaron las identificaciones de las especies y el conteo del número de individuos. Fueron capturados 8,213 individuos del género Lutzomyia, predominando L. ylephiletor (Fairchild \& Hertig) (1097 individuos) siendo también la especie más abundante en Altos de Campana, seguida de L.carpenteri (Fairchild \& Hertig) (610 individuos), y L. dysponeta (Fairchild \& Hertig) (595 individuos). Mediante un análisis de correspondencia simple se relacionaron las especies de Lutzomyia con los tipos de bosques característicos de las áreas de estudios, resultando correspondencia de algunas especies por el tipo de hábitat. En la similitud entre las tres áreas los resultados mostraron que la composición de especies de Phlebotominae en Altos de Campana y Altos Chagres tiene una afinidad de $\mathrm{SSo}=0.503$. Esta investigación también ha permitido actualizar la información ecológica de las especies de interés médico, con el propósito de tomar medidas preventivas y de control para evitar la proliferación de la Leishmaniasis en estas áreas.
\end{abstract}

Palabras clave: Psychodidae, Phlebotominae, Lutzomyia, Distribución, Panamá.

\begin{abstract}
The species of the genus Lutzomyia França are transmitters of the cutaneous leishmaniasis and mucocutaneous in Panama. His distribution is related to the incidence of cases in rural adjacent places to forest, nevertheless the presence of the vectors can register in some areas, without it precence of cases of the illness. This study realized in the localities of Gamboa, Altos de Campana and AltoChagres, during the year 2000 to 2001 in tropical forest in Panamá. The specimens were captured by CDC trap and moved to the Commemorative Institute Gorgas where there were realized the identifications of the
\end{abstract}


species and the count of the number of individuals. There were captured 8,213 individuals of the genus Lutzomyia, L. ylephiletor (Fairchild \& Hertig) (1097 individuals), followed by L.carpenteri (Fairchild \& Hertig) (610 individuals), and L. dysponeta (Fairchild \& Hertig ) (595 individuals). The analysis Correspondence in species of Lutzomyia was related to the types of forests of the areas. In the similarity between three area the results showed that the composition of species of Phlebotominae is presented by Altos de Campana and Alto Chagres $\mathrm{SSo}=0.503$. The investigation has allowed updating the information of some genera and species of medical interesting in the studied area, with the intention of taking preventive measures and of control to avoid the proliferation of the Leishmaniasis in these areas.

Keys Word: Psychodidae, Phlebotominae, Lutzomyia, Distribution, Panamá.

\section{INTRODUCCIÓN}

La Leishmaniasis es una enfermedad parasitaria, de carácter emergente o reemergente de mucha importancia epidemiológica (Ashford 2000), es causante de una alta tasa de morbilidad y mortalidad en más de 88 países en el mundo (Gratz 1999, OMS 1990). Tiene un ciclo epidemiológico que involucra reservorios vertebrados principalmente mamíferos silvestres o caninos domésticos (Herwaldt et al. 1993, Herrer \& Christensen 1976), transmisores invertebrados conocido como chitras en Panamá (Vásquez et al. 1994).

Las chitras son miembros del orden Diptera, y de la familia Psychodidae, subfamilia Phlebotominae. Tienen una amplia distribución en los países americanos de la zona tropical y sub-tropical y su presencia esta relacionada con la incidencia de los casos de leishmaniasis en estas zonas. Existen más de 700 especies en el mundo, en Panamá solo 74 de estas especies se encuentran distribuidas en zonas boscosas (Feliciangeli 1988, Christensen 1972, Herrer \& Christensen 1976), las especies Lutzomyia ylephiletor (Fairchild \& Hertig), L. sanguinaria (Fairchild \& Hertig), L. panamensis (Shannon), L. trapidoi (Fairchild \& Hertig) y L. gomezi (Nitzulescu) han sido incriminadas como vectores de la leishmaniasis tegumentaria en Panamá (Christensen \& Herrer 1973).

La ecología de este vector está relacionada al ambiente selvático, donde realizan su ciclo biológico (Ferro et al. 1998). El riesgo de una exposición puede ocurrir durante la incursión o la realización de actividades recreativas de ecoturismo, científicas, extracción de madera y cacería en las zonas selváticas donde circula el vector y los reservorios (Yadon et al. 2003). Sin embargo, un notable incremento de la tasa de contacto vector-humano ha sido influenciado del impacto ocasionado por el hombre mediante la deforestación de los bosques, cambiando la estructura de las comunidades y el comportamiento de estas chitras, contribuyendo a que aumente la incidencia de transmisión (Duque et al. 2004).

En estos últimos tiempos se ha observado una relevante dominancia de algunas de estas especies en áreas degradadas, cuantificada mediante el índice Alfa de Fisher (Travi 
et al. 2005), indicando que ciertas especies sobreviven, estableciéndose domiciliariamente en casas expuestas permanentemente a la entrada de insectos, sin protección en las ventanas y en su cercanía colinda una vegetación con características apropiadas para permitir el desarrollo del ciclo de vida del vector (Quinnell \& Dye 1994).

El objetivo de este estudio fue identificar la composición de las especies, la similitud en la diversidad de Lutzomyia (cf. Abonnenc \& Leger 1976) y su abundancia relativa presentes en tres localidades de áreas boscosas en Panamá y relacionarlas con sus características ecológicas.

\section{MATERIALES Y MÉTODOS}

Caracterización de las áreas de estudio. Para realizar las colectas de los especimenes se escogieron tres áreas boscosas dentro de la cuenca hidrográfica del Canal de Panamá y cercanas a zonas rurales, allí se ubicaron los puntos de muestreos (Fig.1).

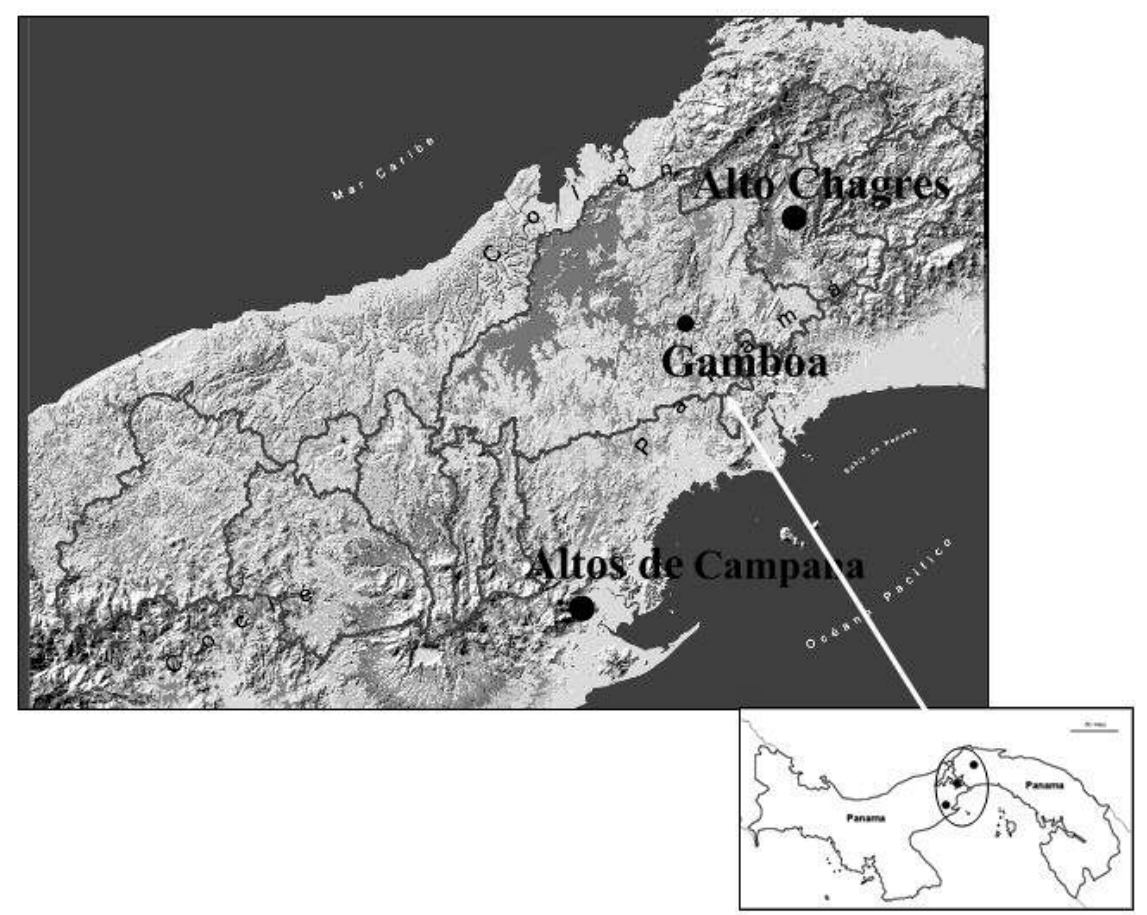

Figura 1. Ubicación de los sitios de estudios Altos de Campana, Gamboa, Alto Chagres, Panamá, Rép. Panamá. 
Valderrama, Herrera \& Salazar: Composición de especies de Lutzomyia França y diferentes tipos de bosques

Área 1: Localizada en El Camino del Oleoducto, próximo al corregimiento de Gamboa, Provincia de Panamá. Su extensión boscosa forma parte del Parque Nacional Soberanía.

Área 2: Localizada en las proximidades de los asentamientos indígenas de la Comarca Woana, en las riveras del Lago Alajuela, dentro del Parque Nacional Alto Chagres.

Área 3: Localizada en el Parque Nacional Altos de Campana, corregimiento de Capira, Provincia de Panamá y muy cercana a la comunidad de Chicá. Una descripción detallada de las condiciones ambientales, geográficas y ecológicas que caracterizan estos tres sitios de muestreos se detalla en el (Cuadro 1).

Cuadro 1. Características geográficas y ecológicas de los sitios de muestreos

\begin{tabular}{|c|c|c|c|}
\hline Variables & $\begin{array}{c}\text { Camino del Oleoducto } \\
\text { (Gamboa) }\end{array}$ & $\begin{array}{l}\text { Alto Chagres } \\
\text { (Colón) }\end{array}$ & $\begin{array}{l}\text { Altos de Campana } \\
\text { (Panamá Este) }\end{array}$ \\
\hline Ubicación & $\begin{array}{c}25 \mathrm{Km} \text {. de la ciudad de } \\
\text { Panamá }\end{array}$ & $\begin{array}{c}40 \mathrm{Km} \text {. de la provincia de } \\
\text { Panamá }\end{array}$ & $\begin{array}{c}60 \mathrm{Km} \text {. de la provincia } \\
\text { de Panamá }\end{array}$ \\
\hline $\begin{array}{l}\text { Coordenadas } \\
\text { geográficas }\end{array}$ & $\begin{array}{c}8^{\circ} 42^{\prime} 59^{\prime \prime} \text { a } 8^{\circ} 54^{\prime} 3^{\prime \prime} \\
\text { Latitud Norte y } \\
81^{\circ} 47^{\prime} 60^{\prime \prime} \text { a } 81^{\circ} 52^{\prime} 56^{\prime \prime} \\
\text { Longitud Oeste }\end{array}$ & $\begin{array}{l}10^{\circ} 11^{\prime} 09^{\prime \prime} \text { a } 10^{\circ} 56^{\prime} 04^{\prime \prime} \\
\text { Latitud Norte y } \\
63^{\circ} 20^{\prime} 02^{\prime \prime} \text { a } 70^{\circ} 40^{\prime} 06^{\prime \prime} \\
\text { Longitud Oeste }\end{array}$ & $\begin{array}{c}8^{\circ} 38^{\prime} 35^{\prime \prime} \text { a } 8^{\circ} 41^{\prime} 30^{\prime \prime} \\
\text { Latitud Norte y } \\
79^{\circ} 53^{\prime} 25^{\prime \prime} \text { a } 80^{\circ} 00^{\prime} 20^{\prime \prime} \\
\text { Longitud Oeste }\end{array}$ \\
\hline Precipitación pluvial & $<2,500 \mathrm{~mm}$ & $\leq 2,200 \mathrm{~mm}$ & $<2,700 \mathrm{~mm}$ \\
\hline Temperatura media & $25^{\circ} \mathrm{C}$ & entre $20^{\circ}$ a $30^{\circ} \mathrm{C}$ & $23^{\circ} \mathrm{C}$ \\
\hline Humedad Relativa & $\pm 80 \%$ & entre los $36-90 \%$ & $90 \%$ \\
\hline
\end{tabular}

Zona de Vida

Tipo de vegetación
Bosque húmedo tropical Bosque húmedo Tropical

Bosque sub perennifolio tropical
Área de cultivos, sabanas y vegetación secundaria Pionera
Bosque muy húmedo tropical premontano

Bosque perennifolio sub tropical 
Diseño experimental, colecta e identificación de los ejemplares. Las colectas se realizaron durante la estación lluviosa desde el 4 de julio del 2000 hasta el 23 de noviembre del 2000 y la estación seca desde el 24 de enero al 1 de junio del 2001. Para colectar los ejemplares, se utilizó el diseño completamente aleatorio, donde se seleccionaron al azar 15 puntos de colecta. A lo largo del todo el período se muestrearon once veces en cada sitio.

Los flebótomos se capturaron con trampas CDC miniatura (Center for Disease Control) (Sudia \& Chamberlain 1962), colocadas a $2 \mathrm{~m}$ de altura, las trampas se dejaron funcionando por 12 horas, iniciando a las 6:00 p.m. y retirándolas a las 6:00 a.m., durante dos días consecutivos. Los ejemplares colectados se preservaron en alcohol glicerado al 70\%. En el laboratorio se procesaron según las técnicas descritas por Forattini (1973) y se identificaron de acuerdo a las claves y características morfológicas de los trabajos de Young \& Duncan (1994).

Análisis de los datos. Se calculó la abundancia relativa de todas las especies colectadas según formula $\mathrm{Pi}=\mathrm{Ni} / \mathrm{N}$ donde $\mathrm{N}$ es la suma total de los individuos de todas las especies presentes en la muestra; $\mathrm{Ni}$ es el número de individuos de la especies.

Además el índice de similitud de Sorensen $[\mathrm{Cs}=2 \mathrm{j} /(\mathrm{a}+\mathrm{b})]$ utilizado para demostrar las similitudes entre las comunidades de flebótomos y los sitios estudiados; donde: $\mathrm{j}=$ número de especies encontradas en ambos estratos, $\mathrm{a}=$ número de especies encontradas en el estrato $\mathrm{A}$ (por ejemplo), y $\mathrm{b}=$ número de especies encontradas en el estrato B (si ese es el estrato de comparación). Los resultados obtenidos de este análisis se interpretan así "si los índices son iguales a 1 hay una perfecta similitud entre estratos y si los índices del área igualan 0 hay una perfecta no similitud" (Magurran 1988).

Los análisis estadísticos se graficaron en un diagrama de Box \& Wisker (Tukey 1977) con la finalidad de hacer un análisis descriptivo de ellos. Los datos de números de individuos de las especies vectores de la leishmaniasis cutánea en Panamá se relacionaron con los tipos de bosques, mediante un análisis de correspondencia simple, utilizando el programa XLSTAT 2006

\section{RESULTADOS}

En las tres áreas seleccionadas en este estudio se colectaron 8,284 individuos de la subfamilia Phlebotominae, un total de 8,213 individuos pertenecen al género Lutzomyia y el $47 \%$ de las especies están relacionados en la transmisión de la leishmaniasis en Panamá.

La abundancia relativa se analizó por especies en cada sitio y se encontró que las especies L. panamensis, L. trapidoi, L. tiramula resultaron estar presente en los tres sitios de muestreos y abundantes en estos sitios. Las especies $L$. dysponeta y $L$. 
carpenteri fueron las más abundantes en Gamboa, L camposi en Gamboa y Altos Chagres, mientras que L. ylephiletor es la más abundante de todas las especies y solo se colecto en Altos de Campana (Cuadro 2). En cuanto al complejo de especies Phlebotominae el Camino del Oleoducto y Altos de Campana son los lugares donde se observó mayor riqueza.

El índice de similitud ha permitido comparar las poblaciones de Lutzomyia que componen estas comunidades y determinar su riqueza en las zonas estudiadas, estableciendo connotaciones importantes en cuanto a la composición de las especies. Según los resultados la similitud entre los sitios de muestreo es poca, sin embargo entre Altos Chagres y Altos de Campana se presenta un valor de $\mathrm{SSo}=0.503$ indicando un poco más afinidad entre estos dos sitios. Los valores de SSo $=0.100$ para Altos de Campana y Gamboa, $\mathrm{SSo}=0.215$ entre Alto Chagres y Gamboa (Cuadro 3)

La Figura 2 presenta los valores promedios, la desviación estándar y el error estándar para cada área estudiada. Se observa que el valor promedio más alto se registra en Gamboa, seguidos por Altos de Campana, mientras que el promedio en Altos Chagres fue bajo. Sin embargo en el mismo grafico se observa que los valores de Altos de Campana y Gamboa presentan mayor desviación del muestreo, que los obtenidos en Altos Chagres, lo cual indica que en Gamboa la mayor cantidad de individuos por especies se encuentran representados en este tipo de bosque.

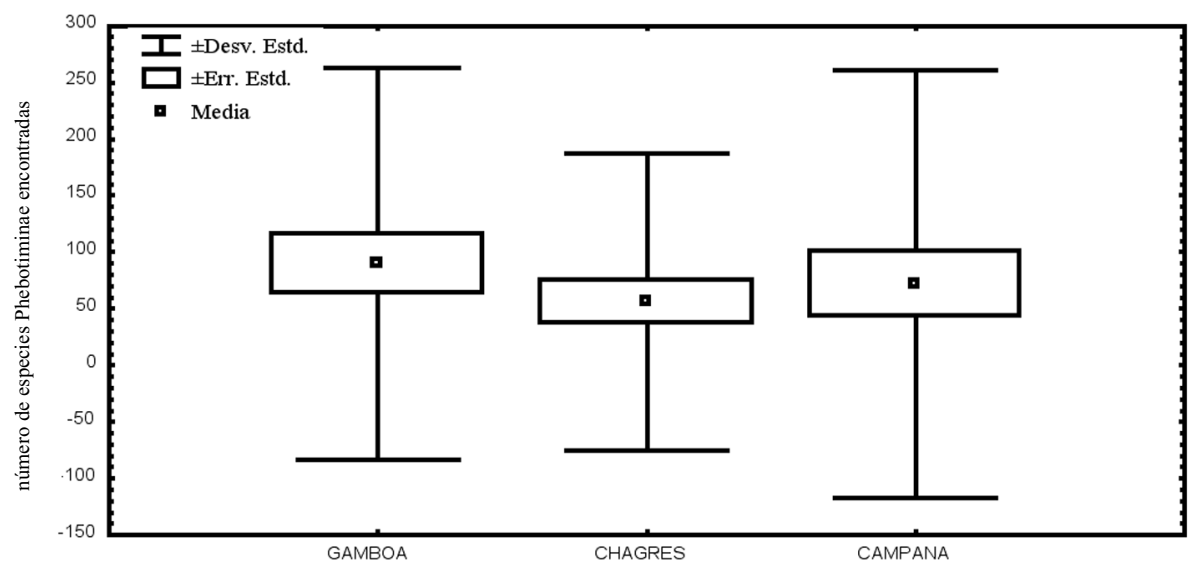

Figura 2. El diagrama de caja muestra el valor promedio (media), la desviación estándar (desv. estd.), el error estandar (err. estd.) de las muestras obtenidas en los sitios seleccionados. 
Cuadro 2. Lista de la especies de Phlebotominae colectadas en trampas CDC y su abundancia relativa $(\mathrm{Pi})$

\begin{tabular}{|c|c|c|c|c|c|c|c|}
\hline \multirow[b]{2}{*}{ ESPECIES } & \multicolumn{2}{|c|}{$\begin{array}{l}\text { Camino del } \\
\text { Oleoducto } \\
\text { (Gamboa) } \\
\end{array}$} & \multicolumn{2}{|c|}{$\begin{array}{l}\text { P.N. Alto } \\
\text { Chagres } \\
\text { (Colón) } \\
\end{array}$} & \multicolumn{2}{|c|}{$\begin{array}{c}\text { P.N. Altos } \\
\text { de Campana } \\
\text { (Panamá Este) } \\
\end{array}$} & \multirow[b]{2}{*}{$\begin{array}{c}\text { Total } \\
\mathrm{x} \text { especies }\end{array}$} \\
\hline & $\mathbf{N}$ & $\begin{array}{c}\mathbf{P i} \\
(\%)\end{array}$ & $\mathbf{N}$ & $\begin{array}{c}\mathbf{P i} \\
(\%)\end{array}$ & $\mathbf{N}$ & $\begin{array}{c}\mathbf{P i} \\
(\%)\end{array}$ & \\
\hline $\begin{array}{l}\text { Brumptomyia hamata } \\
\text { (Fairchild \& Hertig, 1947) } \\
\text { Lutzomvia abonneci }\end{array}$ & 11 & 0.32 & 14 & 0.66 & 8 & 0.29 & 33 \\
\hline $\begin{array}{l}\text { (Floch \& Chassignet, 1947) } \\
\text { Lutzomvia aclydifera }\end{array}$ & 1 & 0.03 & 1 & 0.05 & 0 & 0.00 & 2 \\
\hline (Fairchild \& Hertig, 1952) & 196 & 5.74 & 24 & 1.13 & 29 & 1.06 & 249 \\
\hline Lutzomyia aragaoi (Costa Lima, 1932) & 2 & 0.06 & 2 & 0.09 & 1 & 0.04 & 5 \\
\hline Lutzomyia atroclavata (Knab, 1913) & 0 & 0.00 & 0 & 0.00 & 11 & 0.40 & 11 \\
\hline Lutzomyia barrettoi (Mangabeira, 1941) & 7 & 0.20 & 0 & 0.00 & 5 & 0.18 & 12 \\
\hline $\begin{array}{l}\text { Lutzomyia camposi (Rodríguez, 1952) } \\
\text { Lutzomyia carpenteri }\end{array}$ & 465 & 13.61 & 407 & 19.09 & 68 & 2.49 & 940 \\
\hline (Fairchild \& Hertig, 1953) & 610 & 17.85 & 39 & 1.83 & 36 & 1.32 & 685 \\
\hline Lutzomyia cruciata (Coquillett, 1907) & 0 & 0.00 & 7 & 0.33 & 89 & 3.25 & 96 \\
\hline $\begin{array}{l}\text { Lutzomyia dasymera } \\
\text { (Fairchild \& Hertig, 1961) } \\
\text { Lutzomyia dysponeta }\end{array}$ & 3 & 0.09 & 1 & 0.05 & 1 & 0.04 & 5 \\
\hline (Fairchild \& Hertig, 1952) & 595 & 17.41 & 3 & 0.14 & 17 & 0.62 & 615 \\
\hline Lutzomyia furcata (Mangabeira, 1941) & 3 & 0.09 & 5 & 0.23 & 0 & 0.00 & 8 \\
\hline Lutzomyia gorbitzi (Blancas, 1960) & 2 & 0.06 & 0 & 0.00 & 4 & 0.15 & 6 \\
\hline Lutzomyia geniculata (Mangabeira, 1941) & 6 & 0.18 & 0 & 0.00 & 0 & 0.00 & 6 \\
\hline *Lutzomyia gomezi (Nitzulescu, 1931) & 117 & 3.42 & 31 & 1.45 & 83 & 3.03 & 231 \\
\hline Lutzomyia longipalpis (Lutz \& Neiva, 1912) & 0 & 0.00 & 0 & 0.00 & 1 & 0.04 & 1 \\
\hline Lutzomyia micropyga (Mangabeira, 1942) & 1 & 0.03 & 0 & 0.00 & 0 & 0.00 & 1 \\
\hline Lutzomyia nordestina (Mangabeira 1942) & 66 & 1.93 & 6 & 0.28 & 8 & 0.29 & 80 \\
\hline $\begin{array}{l}\text { Lutzomyia odax (Fairchild \& Hertig, 1961), } \\
\text { Lutzomyia olmeca bicolor }\end{array}$ & 0 & 0.00 & 4 & 0.19 & 4 & 0.15 & 8 \\
\hline (Vargas \& Díaz-Nájera, 1959) & 258 & 7.55 & 30 & 1.41 & 114 & 4.17 & 402 \\
\hline Lutzomyia ovallesi (Ortiz, 1952) & 28 & 0.82 & 0 & 0.00 & 11 & 0.40 & 39 \\
\hline * Lutzomyia panamensis (Shannon, 1926) & 536 & 15.69 & 363 & 17.03 & 312 & 11.41 & 1211 \\
\hline Lutzomyia pessoana (Barretto, 1962) & 4 & 0.12 & 10 & 0.47 & 1 & 0.04 & 15 \\
\hline $\begin{array}{l}\text { Lutzomyia punctigeniculata } \\
\text { (Floch \& Abonnenc, 1944) }\end{array}$ & 3 & 0.09 & 6 & 0.28 & 0 & 0.00 & 9 \\
\hline Lutzomyia insolita (Fairchild \& Hertig, 1956) & 2 & 0.06 & 0 & 0.00 & 0 & 0.00 & 2 \\
\hline Lutzomyia runoides (Fairchild \& Hertig, 1953) & 33 & 0.97 & 27 & 1.27 & 9 & 0.33 & 69 \\
\hline *Lutzomyia sanguinaria (Fairchild \& Hertig, 1957) & 60 & 1.76 & 210 & 9.85 & 275 & 10.05 & 545 \\
\hline Lutzomyia saulensis (Floch \& Abonnenc, 1944) & 4 & 0.12 & 0 & 0.00 & 0 & 0.00 & 4 \\
\hline Lutzomyia serrana (Damasceno \& Arouck, 1949) & 1 & 0.03 & 0 & 0.00 & 3 & 0.11 & 4 \\
\hline Lutzomyia shannoni (Dyar, 1929) & 5 & 0.15 & 1 & 0.05 & 7 & 0.26 & 13 \\
\hline *Lutzomyia trapidoi (Fairchild \& Hertig, 1952) & 148 & 4.33 & 431 & 20.22 & 188 & 6.87 & 767 \\
\hline Lutzomyia trinidadensis (Newstead, 1922) & 11 & 0.32 & 2 & 0.09 & 33 & 1.21 & 46 \\
\hline Lutzomyia triramula (Fairchild \& Hertig, 1952) & 184 & 5.38 & 476 & 22.33 & 256 & 9.36 & 916 \\
\hline Lutzomyia undulata (Fairchild \& Hertig, 1950) & 1 & 0.03 & 0 & 0.00 & 3 & 0.11 & 4 \\
\hline Lutzomyia vesicifera (Fairchild \& Hertig, 1947) & 35 & 1.02 & 15 & 0.70 & 10 & 0.37 & 60 \\
\hline Lutzomyia vespertilionis (Fairchild \& Hertig, 1947) & 15 & 0.44 & 6 & 0.28 & 15 & 0.55 & 36 \\
\hline * Lutzomyia ylephiletor (Fairchild \& Hertig, 1952) & 2 & 0.06 & 11 & 0.52 & 1097 & 40.11 & 1110 \\
\hline Warileya rotundipennis (Fairchild \& Hertig, 1951) & 2 & 0.06 & 0 & 0.00 & 36 & 1.32 & 38 \\
\hline Sub-total & 3417 & & 2132 & & 2735 & & \\
\hline Total & & & & & & & 8284 \\
\hline
\end{tabular}

*Especies de Lutzomyia incriminadas en la transmisión de la leishmaniasis cutánea en Panamá (Christensen et al. 1973). 
Valderrama, Herrera \& Salazar: Composición de especies de Lutzomyia França y diferentes tipos de bosques

Cuadro 3. Índice de Sorensen calculados para las especies de Phlebotominae.

\begin{tabular}{lll}
\hline & Chagres & Gamboa \\
\hline Campana & 0.503 & 0.100 \\
Chagres & & 0.215 \\
\hline
\end{tabular}

En el análisis de correspondencia simple entre los tipos de bosques y las especies de Lutzomyia, explica un 100\% la variabilidad de los datos. El análisis resultó una distribución homogénea indicando que las diversas especies están estrechamente relacionadas al tipo de hábitat estudiado. Las especies de L. trapidoi, L.sanguinaria fueron relacionadas con las características propias de la vegetación de Alto Chagres, L. ylephiletor con Altos de Campana y L. gomezi con Gamboa (Fig.3). Lutzomyia panamensis es una especie que mostró relación con las características de cualquiera de los lugares de estudio.

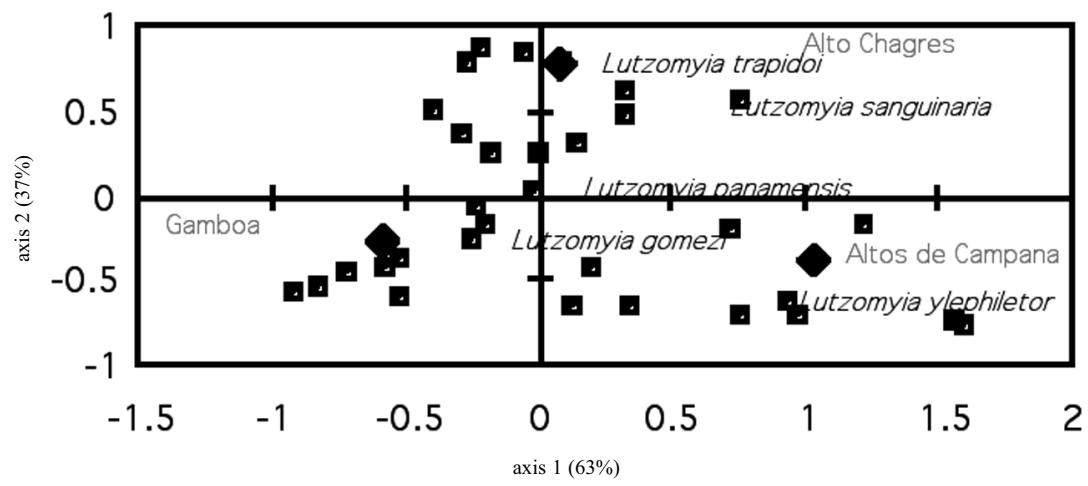

Figura 3. Análisis de correspondencia simple de los bosques ubicados en Gamboa, Altos de Campana, Altos Chagres muestreados desde 2000 al 2001.

Este análisis incluye todas las especies de Lutzomyia colectadas durante este periodo, sin embargo, en el gráfico se muestras las especies de importancia médica. Las especies Lutzomyia trapidoi, L. sanguinaria, L. ylephiletor, L. gomezi y L. panamensis fueron colectadas en las tres áreas de estudio, sin embargo al 
relacionarlas mediante el análisis de correspondencia las especies fueron agrupadas según la cantidad de individuos, estableciéndose la relación con el tipo de bosque o condiciones característico de cada sitio

\section{DISCUSIÓN}

La posición geográfica de Panamá y las características específicas de su clima mantienen ambientes ecológicos variables a los largo del país, que han permitido el establecimiento de muchas especies, presentándose una heterogeneidad en sus comunidades (Hubbell \& Foster 1987, Condit et al. 1992, 1996). Dentro de los bosques tropicales la composición taxonómica vegetativa brinda diversos gradientes que permiten la diversificación de los organismos. (Janzen \& Schoener 1968, Janzen 1987). En este estudio la composición de especies de flebótomos y la abundancia relativa registrada demuestran que esta complejidad ha sido satisfactoria para el desarrollo de esta fauna en los bosques tropicales de Panamá (Chaniotis et al. 1972, Memmott 1992). Sin embargo, la poca afinidad entre los sitios de muestreos y las especies (índice de Sorensen) indica que las diferencias ecológicas y vegetativas son factores determinantes e influyen notablemente en la presencia de algunas especies y en su abundancia poblacional. Según Feitosa \& Castellon (2006) la composición de especies de una población de flebótomos pueden ser influenciada por las condiciones geográficas, meteorológicas, y microclimáticas presentes en determinadas áreas. Las variaciones locales de temperatura y humedad también influyen en la abundancia de los flebótomos (Ostfeld 2004). En el Cuadro 1 se presentan las características de cada tipo de bosques estudiados.

La utilización del método de correspondencia simple en este grupo de insectos establece claramente esta asociación, mediante un análisis más especifico, definiendo a especies más relacionadas a un tipo de bosque, como a otras que pueden estar relacionadas a distintos tipos de bosques. En este análisis nos enfocamos en los resultados obtenidos para las especies incriminadas en la transmisión de la Leishmaniasis, donde L. trapidoi y L. sanguinaria correspondieron a las características y condiciones bióticas encontradas en Altos Chagres; L. ylephiletor fue más asociada a las condiciones y características de Altos de Campana; mientras que L. gomezi con las característica de Gamboa. Sin embargo, la especie $L$. panamensis correspondió a estar más relacionada a las condiciones y características de los tres bosques estudiados. Según estudios realizados por Silva et al. (2007) algunas especies son estrictas al ambiente silvestre, mientras que otras han logrado adaptarse a las modificaciones del hábitat producidas por la influencia del hombre.

La relación de los flebótomos y su hábitat puede estar definida por varios factores, las modificaciones a estas estructuras alteran el equilibrio de las poblaciones (Feitosa 
\& Castellon 2006). La deforestación ha sido una de la causas en ejercer un efecto negativo en la abundancia y diversidad de los flebótomos (Jiménez et al. 2000, Travi et al. 2002). En un enfoque epidemiológico las especies involucradas en la transmisión de patógenos, este desequilibrio permite su migración y adaptarse a las áreas degradadas o estableciéndose en domicilios, aumentando el riesgo a la transmisión de Leishmania sp., que produce la leishmaniasis tegumentaria (Silva et al. 2007).

La cobertura boscosa correspondiente a los sitios seleccionados pertenecen a la Cuenca del Canal de Panamá y los resultados obtenidos en esta investigación no solo aportan información sobre la fauna de flebótomos, si no que también su relación al tipo de vegetación característico en estas áreas. Aunque el manejo de la cuenca contempla un plan de acción para mantener reforestada toda área intervenida, hay que considerar que las alteraciones en la composición vegetal pueden contribuir a la incidencia de la Leishmaniasis (Silva et al. 2007).

\section{AGRADECIMIENTOS}

Al Sr. Roberto Rojas por el apoyo en la captura, montaje y colaboración en la identificación de los ejemplares. Al Dr. Enrique Medianero quién realizo las pruebas de correspondencia simple. A la Agencia de Cooperación Española por otorgarnos los fondos para la realización de esta investigación.

\section{LITERATURA CITADA}

Abonnenc, E. \& N. Leger. 1976. Sur une classification rationelle des diptères Phlebotomidae. Cahiers Off Rech Sci Tech Outre-Mer (Ent Mèd)14: 69-78.

Arias, J.; F. Beltrán \& P. Besjeux. 1996. Epidemiología y control de la leishmaniasis en las Américas, por país o territorio. Cuaderno Técnico n 44 . Organización Panamericana de la Salud. Washington.

Ashford, R. W. 2000. The Leishmaniasis as emerging and reemerging zoonoses. International Journal of Parasitology 30:1269-1281.

Chaniotis, B. N., R. B. Tesh, M. A. Correa \& K. M. Johnson. 1972. Diurnal resting sites of phlebotomine sandflies in a Panamanian tropical forest. Journal of Medical Entomology 9(1):91-98.

Christensen, H. 1972. Check of the Phlebotominae sand flies (Diptera: Psychodidae) of Panama including two species not previously reported. Mosquito News 32 (1). 89.

Christensen, H. \& A. Herrer. 1973. Attractivesness of sentinel animals to vectors of Leishmaniasis in Panamá. American Journal of Tropical Medicine and Hygiene 22:578-584.

Christensen, H., C. Johnson \& A. M. de Vasquez. 1984. Leishmaniasis cutánea en Panamá: un breve resumen. Revista Médica de Panamá. 9(3): 182-1987. 
Condit, R., S. P. Hubbell \& R. B. Foster. 1992. Stability and change of a neotropical moist forest over a decade. Bioscience 42: 822-828.

a shifting climate: results from a 50 ha perman. Changes in a tropical forest with a shifting climate: results from a 50 ha permanent census plot in Panama. Journal Tropical Ecology 12: 231-256.

Duque, P., I. D. Vélez, M. Morales \& D. Sierra. 2004. Sand flies fauna involved in the transmission of cutaneous leishmaniasis in Afro-Colombian and Amerindian Communities of Chocó, Pacific Coast of Colombia. Neotropical Entomology 33:263-4

Feitosa, M. A. C. \& Castellón, E. G. 2006. Fauna de flebotomíneos (Diptera: Psychodidade) em fragmentos de floresta ao redor de conjunto habitacionais na ciudade de Manaus, Amazonas, Brasil. I. Estratificação Vertical. Acta Amazonica. 36(4): 539-548.

Feliciangeli M. D. 1988. La fauna flebotomina (Diptera: Psychodidae) en Venezuela. I. Taxonomia y distribución geográfica. Boletín de la Dirección de Malariología y Saneamiento Ambiental 28: 99-113.

Ferro, C., E. Cárdenas, D. Corredor, A. Morales \& L. E. Munstermann. 1998. Life cycle and fecundity analysis of Lutzomyia shannoni (Dyar) (Diptera: Psychodidae). Memórias do Instituto Oswaldo Cruz 93(2): 195-199.

Forattini O.P. 1973. Entomologia Médica 4. Psychodidae. Phlebotominae. Leishmanioses. Bartonelose. São Paulo: Blücher 1973. 658 pp.

Gratz, N. G. 1999. Emerging and resurging vector-borne disease. Annual Review of Entomology.44:51-75.

Herrer, A. \& H. A. Christensen. 1973. Epidemiological patterns of cutaneous leishmaniasis in Panamá. American Journal of Tropical Medicine and Hygiene 25: 54-58.

in Panama. American Journal of Tropical Medicine and Hygiene 25(1):59-63.

Herwaldt, B.L, S. L. Stokes \& D. Juranek. 1993. American cutaneous leishmaniosis in U.S. travels. Annals of Internal Medicine 118(10):779-784.

Holdridge, L. R. 1979. Ecología basada en Zonas de Vida, Editorial IICA, San José, Costa Rica, 1a. edición.

Hubbell, S. P. \& R. B. Foster. 1987. La estructura espacial a gran escala de un bosque neotropical. Revista de Biología Tropical 35: 7-22.

Janzen, D. H. \& Schoener, T. W. 1968. Differences in abundance and diversity between wetter and drier sities during a tropical dry season. Ecology 49: 96-110.

Janzen, D. H. 1987. Insect diversity of a Costa Rican dry forest: why keep it, and how? Biological Journal of the Linnean Society. 30(4): 343-356.

Jimenez, A. E., J. C. Rojas, F. Vargas, \& M. V. Herrero. 2000. Temporal and spatial variation of Phlebotomine (Diptera: Psychodidae) community diversity in a cutaneous leishmaniasis endemic area of Costa Rica. Journal of Medical Entomology 37(2): 216-221 
Magurran, A. 1988. Diversidad Ecológica y su Medición. Ediciones Vedra S.A. $200 \mathrm{p}$.

Manual de Reforestación Cuenca Hidrográfica del Canal de Panamá Volumen 1. Unidad de Sensores Remotos, Noviembre de 2006

Memmott, J. 1992. Patterns of sandfly distribution in tropical forest: a causal hypothesis. Medical and Veterinary Entomology. 6(3):188-194.

Odum, E. P. 1972. Ecología. México ed. Interamericana. 639 pp.

Organización Mundial de la Salud. 1990. Control of the Leishmaniasis. Technical Report Series 793. Geneva: OMS

Ostfeld, R. S., P. Roy, W. Haumaier, L. Canter, F. Keesing \& E. D. Rowton. 2004. Sand Fly (Lutzomyia vexator) (Diptera: Psychodidae) populations in upstate New York: Abundance, microhabitat, and phenology. Journal of Medical Entomology 41(4)

Quinnell, R. J. \& C. Dye. 1994. An experimental study of the peridomestic distribution of Lutzomyia longipalpis (Diptera: Psychodidae). Bulletin of Entomology Research 84: 379-382.

Silva D. F., R. A. Freitas \& A. M.R. Franco. 2007. Diversidade e abundância de flebotomíneos do gênero Lutzomyia (Diptera: Psychodidae) em áreas de mata do nordeste de Manacapuru, AM. Neotropical Entomology 36(1):138-144

Sudia, W. D. \& R. W. Chamberlain. 1962. Battery operated light trap, an improved model. Mosquitoes News 22: 126-129.

Travi, B. L., G. H. Alder, M. Lozano, H. Cadena \& J. Montoya-Lerma. 2002. Impact of habitat degradation on Phlebotominae (Diptera: Psychodidae) of Tropical Dry Forest in Northern Colombia. Journal of Medical Entomology 39(3): 451-456.

Tukey, J. W. 1977. "Exploratory Data Analysis". Addison Wesley, Reading, MA.

Vásquez, A. M. de, H. Paz, E. Méndez \& J. Alvar. 1994. Leishmaniasis en Panamá. Ministerio de Salud 12 p. Panamá

Yadon, Z. E., L. C. Rodrigues, C. R. Davies \& M. A. Quigley. 2003. Indoor and peridomestic transmisión of American cutaneous Leishmaniasis in nothwester Argentina: A retrospective case control study. American Journal of Tropical Medicine and Hygiene 68 (5): 519-526.

Young, D. G. \& J. A. Arias. 1992. Flebótomos: vectores de Leishmaniasis en las Américas. Organización Panamericana de la Salud, Technical Report $\mathrm{n}^{\circ} 33$.

Young, DG \& Duncan, MA.1994. Guide to the identification and geographic distribution of Lutzomyia sand flies in Mexico, the West Indies, Central and South America (Diptera: Psychodidae). Associated Publisher Florida U.S.A. 